\title{
ON CANONICAL REPRESENTATIONS OF CONVEX POLYHEDRA
}

\author{
DAVID AVIS \\ School Of Computer Science, MGill University, Canada \\ E-mail: avis@cs.mcgill.ca \\ KOMEI FUKUDA \\ School Of Computer Science, MGill University, Canada \\ E-mail: fukuda@cs.mcgill.ca \\ STEFANO PICOZZI \\ Institute of Mathematics, EPFL, Switzerland \\ E-mail: stefano.picozzi@epfl.ch
}

\begin{abstract}
Every convex polyhedron in the Euclidean space $\mathbb{R}^{d}$ admits both H-representation and V-representation. When working with convex polyhedra, in particular largescale ones in high dimensions, it is useful to have a canonical representation that is minimal and unique up to some elementary operations. Such a representation allows one to compare two H-polyhedra or two V-polyhedra efficiently. In this paper, we define such representations that are simple and can be computed in polynomial time. The key ingredients are redundancy removal for linear inequality systems and affine transformations of polyhedra.
\end{abstract}

\section{Introduction}

A convex polyhedron or simply polyhedron in $\mathbb{R}^{d}$ is the set of solutions to a finite system of inequalities with real coefficients in $d$ real variables. For a matrix $A \in \mathbb{R}^{m \times d}$ and a vector $b \in \mathbb{R}^{m}$, a pair $(b, A)$ is said to be an $H$ representation of a convex polyhedron $P$ if $P=\left\{x \in \mathbb{R}^{d} \mid b+A x \geq 0\right\}$. Motzkin's decomposition theorem (see, e.g. ${ }^{4,6}$ ) states that every polyhedron has another representation called a V-representation. For matrices $V \in \mathbb{R}^{p \times d}$ and $R \in \mathbb{R}^{q \times d}$, a pair $(V, R)$ is said to be a $V$-representation of a polyhedron $P$ if $P=\operatorname{conv}(V)+\operatorname{cone}(R)$, where $\operatorname{conv}(M)$ (cone $(M)$, respectively) denotes the convex hull (the nonnegative hull) of the row vectors of the matrix $M$. In each of representations, there are trivial transformations that preserve the represented polyhedron. For an H-representation, each inequality can be multiplied with any positive numbers. For a V-representation, each row of $R$ can be scaled by any positive number. Also, any permutation of inequalities and the row vectors within $V$ and $R$ does not modify the polyhedron. Two representations are considered equal if they are the same up to these

afp CanonicalReprOfPol: submitted to World Scientific on April 22, 20021 
transformations.

One can easily see that neither V- nor H-representations are unique for a given polyhedron. First of all both V- and H-representations can have an unlimited number of redundant vectors or inequalities. Furthermore, even without redundancy, representations are not unique. Namely there are infinite many nonredundant V-representations of polyhedra without extreme points, and infinite many nonredundant H-representation of polyhedra that are not full-dimensional, see Figure 1 . The main objective of the present paper is to

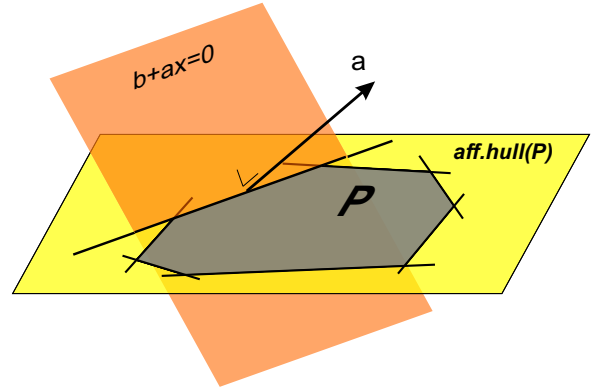

(a)

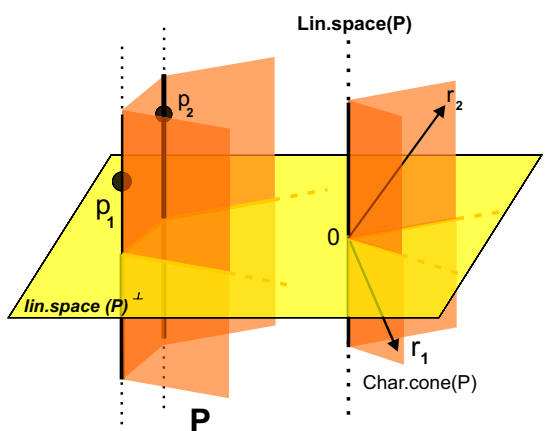

(b)

Figure 1. (a) A 2-dimensional H-polyhedron $P$ in $\mathbb{R}^{3}$, and its affine hull aff $(P)$. One has infinite many possible choices for vector $a$ which preserve the polyhedron $P$. Requiring that $a$ belongs to the linear subspace parallel aff $(P)$ leads to the representation discussed in the book of Schrijver. (b) A 3-dimensional non pointed polyhedron $P=\operatorname{conv}\left(p_{1}, p_{2}\right)+\operatorname{cone}\left(r_{1}, r_{2}\right)+$ lin.space $(P)$, and its characteristic cone char.cone $(P)=$ cone $\left(r_{1}, r_{2}\right)+$ lin.space $(P)$. The $\mathrm{V}$-representation of $P$ is clearly not unique. As discussed by Schrijver, requiring that $p_{1}, p_{2}, r_{1}$ and $r_{2}$ belong to lin.space $(P)^{\perp}$ ensures the uniqueness of the representation.

define a unique V-representation (H-representation, respectively) of a polyhedron that can be computed in polynomial time from any V-representation (H-representation). We shall review the unique polyhedral representations discussed in the book of Schrijver ${ }^{4}$, and propose a variation that is computationally simpler and guaranteed to have certain sparsity property. As a consequence, it is possible to check in polynomial time whether two $\mathrm{H}-$ (or $\mathrm{V}$-) representations define the same polyhedron or not. We also show that redundancy removal is polynomially equivalent to the linear programming. This justifies the use of linear programming algorithms for redundancy removal.

Our main motivation arises from the continuing effort by the first two authors to develop polyhedral computation codes, such as cddlib ${ }^{2}$ and lrslib

afp 'CanonicalReprOfPol: submitted to World Scientific on April 22, 20022 
${ }^{1}$, that perform basic transformations of polyhedral representations. While the main transformation of these codes is the conversion between $\mathrm{H}$ - and Vrepresentations, it is increasing important to make an efficient implementation of algorithms to compute canonical representations. This would allow users to compare two H-polyhedra (or two V-polyhedra) quickly without applying the (often too) expensive conversion. In addition, the computation of a canonical representation can be considered as a preprocessing step for the main transformation, which in some cases results in a drastic speed up.

Due to space limitations, many of the proofs are omitted, and will be included in the full version of the paper.

\section{Representations of Convex Polyhedra}

In this section, we present basic results on representations of convex polyhedra. Most of the results are classical and not very hard to prove. However there is no thorough treatment of this subject for both $\mathrm{V}$ - and $\mathrm{H}$ representations and in particular no study with computational considerations. Throughout this section, $P$ is a non-empty polyhedron in $\mathbb{R}^{d}$.

The notation for H-representation and V-representation given in the last section, although standard, hides the underlying duality of polyhedral cones. A natural way to unify the two representations is through homogenization. We encode a $\mathrm{V}$-representation $(V, R)$ as a pair of a vector and a matrix $\left(\left[\begin{array}{l}e \\ 0\end{array}\right],\left[\begin{array}{l}V \\ R\end{array}\right]\right)$, where $e$ is the column vector of all 1 's. In this representation written as $(b, A)$, each row index $i$ with $b_{i}=1$ indicates that the correspond$\operatorname{ing} i^{\text {th }}$ row vector $A_{i}$ of $A$ is a convex hull generator, and each $b_{i}=0$ indicates $A_{i}$ is a cone generator. This way, both representations are of the same form. Actually we must go even further to define a unique representation of any given polyhedron, in both $\mathrm{V}$ and $\mathrm{H}$ formats.

Let $A$ be an $m \times d$ matrix, $b$ an $m$-dimensional vector and $\{I, L\}$ be a partition of $[m]:=\{1, \ldots, m\}$. We define a quadruple $(b, A, I, L)$ to be an H-representation of the polyhedron

$$
P_{H}=\left\{x \in \mathbb{R}^{d} \mid b_{I}+A_{I} x \geq \mathbf{0}, b_{L}+A_{L} x=\mathbf{0}\right\} .
$$

We define a quadruple $(b, A, I, L)$ to be a V-representation of the polyhedron

$$
P_{V}=\left\{x \in \mathbb{R}^{d} \mid x=A^{T} y, y_{I} \geq \mathbf{0}, b^{T} y=1\right\} .
$$

The set $L$ is called the linearity of the representation. Two representations $(b, A, I, L)$ and $\left(b^{\prime}, A^{\prime}, I^{\prime}, L^{\prime}\right)$ are said to be equivalent if the represented polyhedra are equal. Each row index $i \in[m]$ is called redundant in a representation

afp CanonicalReprOfPol: submitted to World Scientific on April 22, 20023 
$(b, A, I, L)$ if the represented polyhedron stays unchanged after deleting the $i^{\text {th }}$ data, namely removing $b_{i}, A_{i}, i$ from $I$ or $L$ whichever contains $i$, and shifting the rest of the data accordingly. A representation is nonredundant if it has no redundant row index. For technical reasons, if the zero vector appears in a V-representation it will be considered non redundant.

A V-representation $(b, A, I, L)$ is called standard if $b$ is binary (i.e. $b_{i} \in$ $\{0,1\})$ and $b_{L}=\mathbf{0}$. A V-representation can be transformed to an equivalent standard V-representation in quadratic time. For this reason, we assume for the rest of the paper that any V-representation is standard. Each vector $A_{i}$ in a representation is called a generator. It is a point generator if $b_{i}=1$, a ray generator if $b_{i}=0$ and $i \notin L$, and a line generator if $b_{i}=0$ and $i \in L$.

We say two representations $(b, A, I, L)$ and $\left(b^{\prime}, A^{\prime}, I^{\prime}, L^{\prime}\right)$ equal if $b_{L}+$ $A_{L} x=0 \Leftrightarrow b_{L^{\prime}}+A_{L^{\prime}} x=0$ and if there is a permutation $\pi$ of $I$ such that $\pi(I)=I^{\prime}$ and each $\left(b_{i}^{\prime}, A_{i}^{\prime}\right)$ is a positive multiple of $\left(b_{\pi(i)}, A_{\pi(i)}\right)$ for any $i \in I$. Clearly, if two representations of the same type are equal, they are equivalent and thus define the same polyhedron. In particular for V-representation, $b_{L}=0$ and then the first equivalence coincides with the statement $\{x \in$ $\left.\mathbb{R}^{d} \mid x=\left(A_{L}\right)^{T} y\right\}=\left\{x \in \mathbb{R}^{d} \mid x=\left(A_{L^{\prime}}\right)^{T} y\right\}$.

While the equivalence of two representations is nontrivial to check, the equality is easy to check using Gaussian elimination. Our main goal is to discuss an efficient procedure to reduce any representation to a uniquely defined equivalent representation. In particular, we want to extend the definition of the representation discussed in the book of Schrijver ${ }^{4}$ in order to get a canonical representation guaranteeing a certain sparsity property. Extending this definition uses the notion of compatible and complementary linear subspaces of $\mathbb{R}^{d}$.

\subsection{Compatible and Complementary linear subspaces}

We say two linear subspaces $S_{1}$ and $S_{2}$ of $\mathbb{R}^{d}$ having same dimension are compatible if the orthogonal projection of $S_{1}$ onto $S_{2}$ is $S_{2}$ itself, or, equivalently, if the orthogonal projection of $S_{2}$ onto $S_{1}$ is $S_{1}$ itself. We say two linear subspaces $S_{1}$ and $S_{2}$ of $\mathbb{R}^{d}$ are complementary if any basis of $S_{1}$ and any basis of $S_{2}$ form, together, a basis of $\mathbb{R}^{d}$. It follows from elementary linear algebra that $S_{1}$ and $S_{2}$ are complementary if and only if $\operatorname{dim}\left(S_{1}\right)+\operatorname{dim}\left(S_{2}\right)=d$ and $S_{1} \cap S_{2}=\{\mathbf{0}\}$. Similarly any $r \in \mathbb{R}^{d}$ can be written uniquely as $r=r_{1}+r_{2}$, where $r_{1} \in S_{1}$ and $r_{2} \in S_{2}$. For any two complementary linear subspaces $S_{1}$ and $S_{2}$ of $\mathbb{R}^{d}$, we let $\mathcal{P}_{S_{1} S_{2}}$ denote the projector onto $S_{1}$ along $S_{2}$ defined as

$$
\begin{aligned}
\mathcal{P}_{S_{1} S_{2}}: \mathbb{R}^{d} & \longrightarrow S_{1} \\
r & \longmapsto \mathcal{P}_{S_{1} S_{2}}(r)=r_{1} .
\end{aligned}
$$

afp CanonicalReprOfPol: submitted to World Scientific on April 22, 20024 
It is easy to see that $\mathcal{P}_{S_{1} S_{2}}$ is a projector in the sense of the linear algebra. By linearity, this definition can be extended to any subset of $\mathbb{R}^{d}$.

\subsection{Canonical V-Representation}

To define a unique V-representation of a general (non-pointed) polyhedron we need a few more definitions. The linearity space lin.space $(P)$ of $P$ is defined as

$$
\text { lin.space }(P)=\left\{z \in \mathbb{R}^{d} \mid x+\alpha z \in P \text { for all } x \in P \text { and } \alpha \in \mathbb{R}\right\} .
$$

The characteristic cone char.cone $(P)$ of $P$ is

$$
\text { char.cone }(P)=\left\{z \in \mathbb{R}^{d} \mid x+\alpha z \in P \text { for all } x \in P \text { and } \alpha \geq 0\right\} \text {. }
$$

The following defines the V-representation discussed in the book of Schrijver: Lemma 2.1. A nonredundant $V$-representation $(b, A, I, L)$ of polyhedron $P$ satisfying the following exists and is unique:

(1) lin.space $(P)=\left\{z \in \mathbb{R}^{d} \mid z=\left(A_{L}\right)^{T} y_{L}\right\}$, and

(2) all row vectors $A_{i}(i \in I)$ are orthogonal to lin.space $(P)$.

This lemma gives a theoretically satisfactory definition of the canonical representation. Its weakness is that it tends to make the resulting representation dense. The next theorem gives more general criteria which allows one to look for unique $\mathrm{V}$-representations satisfying certain sparsity properties.

Theorem 2.2. A nonredundant $V$-representation $(b, A, I, L)$ of polyhedron $P$ satisfying the following exists and is unique:

(1) lin.space $(P)=\left\{z \in \mathbb{R}^{d} \mid z=\left(A_{L}\right)^{T} y_{L}\right\}$, and

(2) all row vectors $A_{i}(i \in I)$ are orthogonal to $S$,

where $S$ is any fixed subspace of $\mathbb{R}^{d}$ compatible with lin.space $(P)$.

The V-representation of Lemma 2.1 is the special case of Theorem 2.2 when $S$ is chosen to be lin.space $(P)$ itself.

\subsection{Canonical H-Representation}

In this section, we use the notion of compatible spaces to find a general canonical H-representation. We denote by aff $(P)$ the affine hull of $P$. The Hrepresentation discussed in the book of Schrijver is:

Lemma 2.3. A nonredundant $H$-representation $(b, A, I, L)$ of a nonempty polyhedron satisfying the following exists and is unique:

afp CanonicalReprOfPol: submitted to World Scientific on April 22, 20025 
(1) $\operatorname{aff}(P)=\left\{x \in \mathbb{R}^{d} \mid b_{L}+A_{L} x=\mathbf{0}\right\}$, and

(2) each row of $A_{I}$ is the linear space parallel to aff $(P)$.

Again this representation might be computationally inconvenient. The next theorem gives more flexible notion of canonical H-representations which allows one to look for those satisfying additional favourable properties such as sparsity.

Theorem 2.4. A nonredundant $H$-representation $(b, A, I, L)$ of polyhedron $P$ satisfying the following exists and is unique:

(1) $\operatorname{aff}(P)=\left\{x \in \mathbb{R}^{d} \mid b_{L}+A_{L} x=\mathbf{0}\right\}$, and

(2) each row of $A_{I}$ is in $S$,

where $S$ is any linear subspace compatible with the linear space parallel to aff $(P)$.

\section{Computing Canonical Representations}

Given any V- (resp. H-) representation $(b, A, I, L)$ of a polyhedron $P$ in $\mathbb{R}^{d}$, our goal is to compute efficiently the unique equivalent representation $\left(b^{\prime}, A^{\prime}, I^{\prime}, L^{\prime}\right)$ satisfying the following :

(1) it is nonredundant;

(2) lin.space $(P)=\left\{x \in \mathbb{R}^{d} \mid x=\left(A_{L^{\prime}}^{\prime}\right)^{T} y\right\}$ (resp. aff $(P)=\left\{x \in \mathbb{R}^{d} \mid b_{L^{\prime}}^{\prime}+\right.$ $\left.\left.A_{L^{\prime}}^{\prime} x=0\right\}\right)$

(3) the rows of $A_{I^{\prime}}^{\prime}$ are orthogonal to a subspace $S$ compatible with lin.space $(P)$ (resp. are contained in a subspace $S$ compatible with $\mathcal{P}_{0}$ ).

Let $\bar{P}_{0}$ denote lin.space $(P)$ in V-format, and aff $(P)$ in H-format, and let $\bar{S}$ denote $S^{\perp}$ in $\mathrm{V}$-format, and $S$ in H-format. Then, (3) can be replaced by :

(3') the rows of $A_{I^{\prime}}^{\prime}$ are the projections onto $\bar{S}$ along $\bar{P}_{0}$ of any equivalent set of generators of $P$.

We now discuss two special choices for the linear subspace $S$.

Any linear subspace is compatible with itself. Choosing $\bar{S}$ as $\bar{P}_{0}$ itself leads to the orthogonal representation discussed in the book of Schrijver ${ }^{4}$. In this case, the projector $\mathcal{P}_{\bar{S}} \bar{P}_{0}$ onto $\bar{S}$ along $\bar{P}_{0}$ coincides with the orthogonal projector $\mathcal{P}_{\bar{S}}$ onto $\bar{S}$. This representation is perhaps mathematically the most natural but it tends to lead to a very dense representation, (see e.g. Section 6).

afp 'CanonicalReprOfPol: submitted to World Scientific on April 22, 20026 
Let us consider another natural choice for $S$. A coordinate subspace is any vector subspace of $\mathbb{R}^{d}$ generated by some unit vectors $e^{j}(j=1, \ldots, d)$. Selecting $\bar{S}$ as a coordinate subspace guarantees a certain sparsity of the projected vectors. Moreover, selecting $\bar{S}$ as the lexicographically smallest subspace complementary with $\left(\bar{P}_{0}\right)$ guarantees that the nonzero coordinates are indexed by indices as small as possible. The resulting representation will be called the lexicographically smallest or lexico-smallest representation.

Computationally, the orthogonal representation requires the computation of orthogonal basis of a linear subspace, which amounts to doing the Gram-Schmidt orthogonalization procedure. The lexico-smallest representation needs only to check whether a unit $e^{j}$ is linearly independent of a given set of chosen vectors, which amounts to applying Gaussian eliminations.

\section{Redundancy Removal}

\subsection{Redundancy Removal in V-representation}

The techniques used for solving redundancy removal are very similar in both $\mathrm{V}$ and $\mathrm{H}$ formats. Therefore, the discusssion in $\mathrm{H}$-representation will be omitted. A complete description of the redundancy removal techniques in both $\mathrm{H}-$ and V-respresentation can be found in the Polyhedral Computation FAQ ${ }^{3}$ of K. Fukuda.

For any given $\mathrm{V}$-representation $(b, A, I, L)$ of a polyhedron $P$, we let $I_{0}=$ $\left\{i \in I \mid b_{i}=0\right\}$ and $I_{1}=\left\{i \in I \mid b_{i}=1\right\}$. It follows from the definitions, that $P=\operatorname{conv}\left(A_{I_{1}}\right)+\operatorname{cone}(R)$, where $R:=\left[\begin{array}{l}A_{I_{0}} \\ A_{L}^{ \pm}\end{array}\right]$and $A_{L}^{ \pm}:=\left[\begin{array}{c}+A_{L} \\ -A_{L}\end{array}\right]$. Let $(b, A, I, L)$ be any $\mathrm{V}$ representation of polyhedron $P$. The definitions of point, ray and line generators of $P$ imply that,

(1) $k \in I_{0}$ is redundant if and only if $A_{k}$ is redundant in cone $(R)$;

(2) $k \in L$ is redundant if and only if $A_{k}$ and $-A_{k}$ are redundant in cone $(R)$.

(3) $k \in I_{1}$ is redundant if and only if $A_{k}$ is redundant in $\operatorname{conv}(V)+\operatorname{cone}(R)$. For any row index $k \in I \cup L$, we define the linear program R.LPV $\left(b_{k}, A_{k}\right)$ as

$$
\begin{array}{lll}
\max 0 & \\
\text { s.t. } & \left(A_{k}\right)^{T}=b_{k} \sum_{i \neq k}\left(A_{i}\right)^{T} x_{i}+\sum_{l \neq k}\left(R_{l}\right)^{T} y_{l}, & i \in I_{1}, \quad l \in I_{R} \\
& b_{k}=b_{k} \sum_{i \neq k} x_{i}, & i \in I_{1} \\
& x_{i} \geq 0, \quad y_{l} \geq 0, & \forall i \in I, \quad \forall l \in I_{R}
\end{array}
$$

where $I_{R}$ is the index set of the rows of matrix $R$.

Theorem 4.1. Let $(b, A, I, L)$ be any $V$-representation $P$. Then,

(1) $k \in I$ is redundant if and only if $R . L P V\left(b_{k}, A_{k}\right)$ has optimal value zero,

afp CanonicalReprOfPol: submitted to World Scientific on April 22, 20027 
(2) $k \in L$ is redundant if and only if both $R \cdot L P V\left(0, A_{k}\right)$ and $R \cdot L P V\left(0,-A_{k}\right)$ have optimal value zero.

Removing sequentially the redundancies from any representation ( $b, A, I, L)$ of a polyhedron $P$ using this theorem leads to a nonredundant representation $\left(b^{\prime}, A^{\prime}, I^{\prime}, L^{\prime}\right)$ in $\mathcal{O}(m \times \operatorname{LP}(m, d))$ time, where $m=|I \cup L|$. The size of the LPs can be reduced to that of the resulting nonredundant system by using the techniques given by Ottmann et al. ${ }^{5}$

\subsection{Computing lin.space $(P)$ and aff $(P)$}

The canonical V- (resp. H-) representation of polyhedron $P$ is defined so that the rows of the matrix $A_{L}$ form a basis of $S\left(A_{L}\right)$. In this section, we present how one can decide efficiently whether any given row index $k \in I \cup L$ from any nonredundant representation $(b, A, I, L)$ of $P$ belongs to the linearity of the canonical representation of $P$. Solving this decision problem for every $k \in I \cup L$ leads to a set of indices, say $L^{\prime \prime}$, such as $\left(A_{L^{\prime \prime}}\right)^{T} y_{L} \in$ lin.space $(P)$ (resp. such as $\left.b_{L^{\prime \prime}}+A_{L^{\prime \prime}} x=\mathbf{0}\right)$. The linearity $L^{\prime}$ of the canonical representation of $P$ then arises from $L^{\prime \prime}$ by removing the redundancies.

This decision problem can be easily reduced to linear programming. But it may also be (equivalently) solved using the redundancy recognition. This latter method has a nice and simple geometric interpretation in both $\mathrm{H}$ and $\mathrm{V}$ formats.

We first consider V-representations. Clearly, we only have to discuss the case of the ray generators of $P$. Any ray generator $A_{k}$ of $P$ belongs to lin.space $(P)$ if and only if both $A_{k}$ and $-A_{k}$ belong to lin.space $(P)$. In other words,

Theorem 4.2. Let $(b, A, I, L)$ be any $V$-representation of polyhedron $P=$ $\operatorname{conv}\left(A_{I_{1}}\right)+\operatorname{cone}(R)$, where $I_{1}$ and $R$ are defined as in Section 4.1. Then, any row $A_{k}$ of matrix $A$ belongs to lin.space $(P)$ if and only if $k \in L \cup L_{I}$, where $L_{I}=\left\{i \in I_{0} \mid A_{k}\right.$ is redundant in cone $\left.(-R)\right\}$.

Now, consider H-representations. One has to decide whether $\left\{x \in \mathbb{R}^{d} \mid b_{k}+\right.$ $\left.A_{k} x=0\right\} \supseteq$ aff $(P)$. It is clearly the case if and only if $P \subseteq\left\{x \in \mathbb{R}^{d} \mid b_{k}+\right.$ $\left.A_{k} x=0\right\}$. In other words,

Theorem 4.3. Let $(b, A, I, L)$ be any H-representation of polyhedron $P$. Then, any row $A_{k}$ of matrix $A$ is such as $\left\{x \in \mathbb{R}^{d} \mid b_{k}+A_{k} x=0\right\} \supseteq$ aff $(P)$ if and only if $k \in L \cup L_{I}$, where $L_{I}=\left\{i \in I \mid-b_{i}-A_{i} x \geq 0\right.$ is redundant with $\left.\left\{b_{I}+A_{I} x \geq 0\right\} \cup\left\{b_{L}+A_{L} x=0\right\}\right\}$.

afp CanonicalReprOfPol: submitted to World Scientific on April 22, 20028 


\section{Computational Equivalence of Problems}

The results of the last section show how a canonical representation may be computed efficiently. The following theorem, which directly follows from the preceding results, characterizes the complexity of this computation.

Theorem 5.1. Redundancy removal and the computation of a canonical representation are polynomially equivalent problems.

As we saw in Section 4, redundancy removal has complexity $\mathcal{O}(m \times \operatorname{LP}(m, d))$, where $m=|I \cup L|$. Solving these linear programs is expensive in time. The natural question that arises concerns the necessity of solving linear programs to remove redundancies. It is well known that checking feasibility of a linear system is polynomially equivalent to linear programming. The following lemma then states the polynomial equivalence of redundancy removal and the linear programming.

Lemma 5.2. Let $A x+b \geq \mathbf{0}$, where $A \in \mathbb{R}^{m \times d}$ and $b \in \mathbb{R}^{d}$, be any linear system of inequalities. Then, $A x+b \geq \mathbf{0}$ is feasible if and only if the inequality $\left\{x_{0} \leq 1\right\}$ is not redundant with $A x+b x_{0} \geq \mathbf{0}$.

Proof. Consider the homogenized system $A x+b x_{0} \geq \mathbf{0}$. Clearly, $A x+b \geq \mathbf{0}$ is feasible if and only if it exist a vector $\bar{x} \in \mathbb{R}^{d}$ such that the pair $\left(x_{0}=\right.$ $1 ; x=\bar{x})$ is a feasible solution of $b x_{0}+A x \geq \mathbf{0}$. This is the case if and only if $\left(x=\alpha \bar{x} ; x_{0}=\alpha\right)$ is a feasible solution of $b x_{0}+A x \geq \mathbf{0}$ for any $\alpha \geq 0$. In particula, $b+A x \geq \mathbf{0}$ is feasible if and only if it exist an $x \in \mathbb{R}^{d}$ such that $\left(x ; x_{0}>1\right)$ is a feasible solution of $b x_{0}+A x \geq \mathbf{0}$. On the other hand, the system $b x_{0}+A x \geq \mathbf{0}, x_{0} \leq 1$ is feasible. Then, one may check whether the inequality $\left\{x_{0} \leq 1\right\}$ is redundant with $b x_{0}+A x \geq \mathbf{0}$ or not. In particular, it is not hard to see that $b x_{0}+A x \geq 0, x_{0}>1$ is feasible if and only if $\left\{x_{0} \leq 1\right\}$ is redundant with $b x_{0}+A x \geq \mathbf{0}$.

It follows from the above results that we may check the feasibility of any linear system $A x \leq b$ in polynomial time by performing redundancy removal. As a direct consequence one has the argued result that,

Theorem 5.3. Redundancy removal, the computation of a canonical representation and linear programming are polynomially equivalent problems.

\section{Some Examples}

We start by illustrating the difference between the two canonical representations described in Section 6. Consider the following $(b, A, I, L) \mathrm{V}$ -

afp CanonicalReprOfPol: submitted to World Scientific on April 22, 20029 
representation:

$$
b=\left[\begin{array}{l}
1 \\
1 \\
1
\end{array}\right], A=\left[\begin{array}{llllll}
0 & 1 & 1 & 1 & 1 & 0 \\
1 & 0 & 1 & 1 & 0 & 1 \\
1 & 1 & 0 & 0 & 1 & 1
\end{array}\right], I=\{1,2,3\}, L=\emptyset .
$$

(This corresponds to the three hamiltonian circuits for the complete graph on four vertices.) An orthogonal H-representation in this case is given by:

$$
b=\left[\begin{array}{l}
-2 \\
-2 \\
-2 \\
-2 \\
1 / 3 \\
1 / 3 \\
1 / 3
\end{array}\right], A=\left[\begin{array}{cccccc}
1 & 1 & 1 & 0 & 0 & 0 \\
1 & 0 & 0 & 1 & 1 & 0 \\
0 & 1 & 0 & 1 & 0 & 1 \\
0 & 0 & 1 & 0 & 1 & 1 \\
-1 / 3 & 1 / 6 & 1 / 6 & 1 / 6 & 1 / 6 & -1 / 3 \\
1 / 6 & -1 / 3 & 1 / 6 & 1 / 6 & -1 / 3 & 1 / 6 \\
1 / 6 & 1 / 6 & -1 / 3 & -1 / 3 & 1 / 6 & 1 / 6
\end{array}\right], I=\{5,6,7\}, L=\{1,2,3,4\}
$$

Note that apart from the linearity space, the matrix is completely dense. The lexico-smallest H-representation is given by:

$$
b=\left[\begin{array}{c}
-2 \\
-2 \\
-2 \\
-2 \\
1 \\
1 \\
-1
\end{array}\right], A=\left[\begin{array}{cccccc}
1 & 1 & 1 & 0 & 0 & 0 \\
1 & 0 & 0 & 1 & 1 & 0 \\
0 & 1 & 0 & 1 & 0 & 1 \\
0 & 0 & 1 & 0 & 1 & 1 \\
-1 & 0 & 0 & 0 & 0 & 0 \\
0 & -1 & 0 & 0 & 0 & 0 \\
1 & 1 & 0 & 0 & 0 & 0
\end{array}\right], I=\{5,6,7\}, L=\{1,2,3,4\} .
$$

This polytope is two dimensional, and so the second representation is quite sparse and contains a square 3 by 4 submatrix of zeroes in the rows indexed by $I$. (These inequalities have the natural interpretation $x_{12} \leq 1, x_{13} \leq$ $1, x_{12}+x_{13} \geq 1$ for the travelling salesman problem).

Redundancy removal can have a significant effect in speeding up the running time of vertex enumeration algorithms. This is particularly pronounced on algorithms which enumerate bases, such as pivot based algorithms, and other algorithms that use symbolic perturbation to resolve degeneracy. However improvement is also noticeable for double description algorithms. Here we give a few examples, using $\operatorname{lrs}^{1}$ as an example of a pivot based method, and cdd $^{2}$ as an example of a double description based method.

Consider the metric cone which for any integer $n \geq 3$ is a polyhedron in $R^{n(n-1) / 2}$ defined by the triangle inequalities $x_{i j}-x_{i k}-x_{j k} \geq 0$ for all distinct $1 \leq i, j, k \leq n$, where $x=\left(x_{i j}\right) 1 \leq i<j \leq n$. The metric cone is extremely degenerate. Vectors $\mathrm{x}$ satisfying these inequalities are known as semimetrics: they are nonnegative and satisfy all triangle inequalities. Often the nonnegativity condition $x_{i j} \geq 0$ is explicitly specified although these inequalities are in fact redundant. The program lrs has a nonnegative option for specifying these additional inequalities, but using it here causes a big increase in the running time. For example, with $n=6$ and without the redundant constraints, lrs generates 203,956 bases to find the 296 extreme rays. With the nonnegative

afp CanonicalReprOfPol: submitted to World Scientific on April 22, 200210 
option, it generates $1,960,411$ bases and so the running time is nearly 10 times longer. The effect on cdd is not so pronounced but still noticeable: including the redundant inequalities nearly doubles the computation time.

An other way redundancy occurs is when the programs are used to generate rays lying on lower dimensional faces. This is easily performed in both lrs and cdd by including a linearity option which specifies that certain inequalities should be treated as equations. This normally results in some of the original inequalities becoming redundant. Taking one such linearity causes all rays on a facet to be generated. For the metric cone with $n=6$, a facet contains 113 extreme rays. Without removing redundant inequalities, lrs generates 121,215 bases. There are 14 redundant inequalities, and after there removal lrs generates 38,119 bases, a speed up of a factor of about 4 . Choosing two linearities causes ray enumeration on a ridge. Here the effect of redundancy removal is much more pronounced, as 30 of the original constraints are redundant. lrs runs more than 50 times faster after redundancy removal. The effect of redundancy removal on cdd is also noticeable, with speed ups of the order of about $31 / 2$ and 6 times respectively.

\section{References}

1. D. Avis. lrs Homepage, 2001. School of Computer Science, McGill University, Canada. http://cgm.cs.mcgill.ca/ ${ }^{\sim}$ avis/C/lrs.html.

2. K. Fukuda. cddlib reference manual, cddlib Version 092a.

Swiss Federal Institute of Technology, Switzerland, 2001. http://www.ifor.math.ethz.ch/ fukuda/cdd_home/cdd.html.

3. K. Fukuda. Frequently Asked Questions in Polyhedral Computation Swiss Federal Institute of Technology, Switzerland, 2000 http://www.ifor.math.ethz.ch/ffukuda/polyfaq/polyfaq.html

4. A. Schrijver. Theory of Linear and Integer Programming. John Wiley \& Sons, New York, 1986.

5. Th. Ottmann and S. Schuierer and S. Soundaralakshmi. Enumerating extreme points in higher dimensions. STACS 95: 12th Annual Symposium on Theoretical Aspects of Computer Science. Springer-Verlag,1995.

6. G.M. Ziegler. Lectures on polytopes. Graduate Texts in Mathematics 152. Springer-Verlag, 1994.

afp CanonicalReprOfPol: submitted to World Scientific on April 22, 200211 